
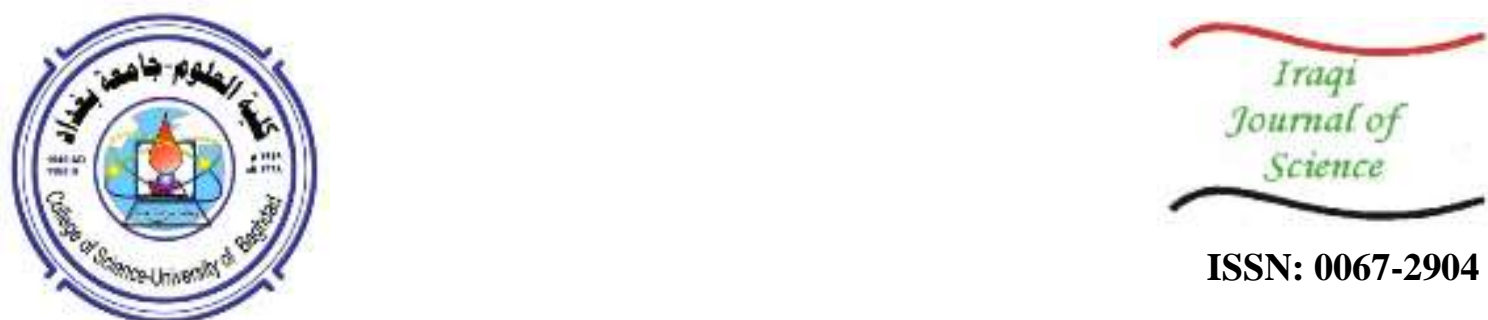

ISSN: 0067-2904

\title{
Fourier Transform Coding-based Techniques for Lossless Iris Image Compression
}

\author{
Ghadah Al-Khafaji, Noor Ali
}

Department of Computer Science, University of Baghdad, College of Science

\begin{abstract}
Today, the use of iris recognition is expanding globally as the most accurate and reliable biometric feature in terms of uniqueness and robustness. The motivation for the reduction or compression of the large databases of iris images becomes an urgent requirement. In general, image compression is the process to remove the insignificant or redundant information from the image details, that implicitly makes efficient use of redundancy embedded within the image itself. In addition, it may exploit human vision or perception limitations to reduce the imperceptible information.

This paper deals with reducing the size of image, namely reducing the number of bits required in representing the image. This was performed by exploiting the transforms-based coding techniques of lossless base compression system. In these techniques, the first part looked at the traditional Fourier transform coding technique while the second part aimed at enhancing the performance of the traditional transformation techniques. This was achieved once by overcoming the inherited problems of this technique that suffers from the complex nature base, then latter by incorporating the double base coding techniques of hierarchal scheme, as mixing of both discrete wavelet transform and zipper coding techniques.

The test results indicated that the proposed scheme produced high compression ratio with identically preserving the quality of the compressed (decoded) image.
\end{abstract}

Received: 15/5/2019

Accepted: 17/7/2019

Keywords: decoded image, zipper

\section{تحويل فورير (Fourier) تقنية قائمة على الترميز لضغط صورة القزحية بدون فقدان للبيانات}

\author{
غادة كاظم طعمة، نور علي جليع \\ قسم الحاسبات، كلية العلوم، جامعة بغداد، بغداد، العراق فئه
}

الخلاصة

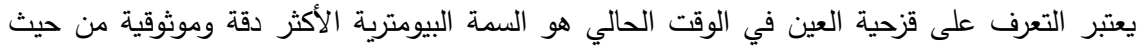



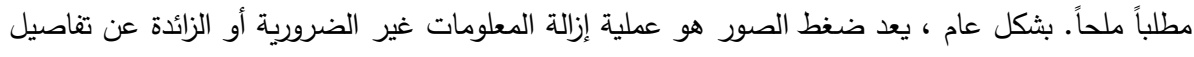

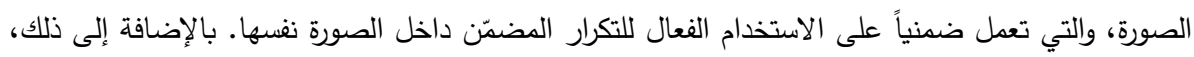



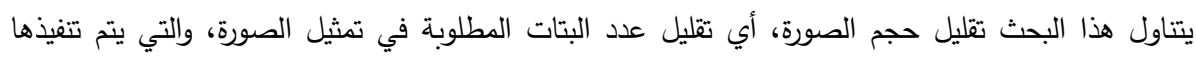

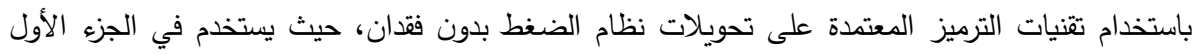

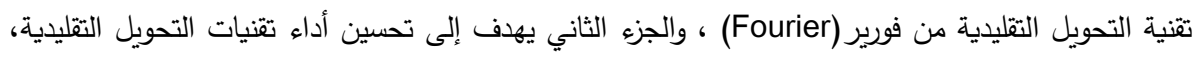

بواسطة التغلب على المشاكل الموروثة لهذه التقنية التي تعاني من طبيعتها المعقدة، ثم الجزء الأخير من خلال 




\section{Introduction}

Iris recognition system for identifying authentication and verification is one of the most precise and accepted biometrics that is gaining popularity in the world. The iris is the colored portion of the eye surrounding the pupil, contains unique patterns which are prominent under near-infrared illumination. These patterns remain stable from a very young age, barring trauma or disease, allowing accurate identification with a very high level of confidence [1].

The enrolment of an individual into a commercial iris system requires capturing one or more images from a video stream. Typically, the database for such system does not contain actual iris images, but rather it stores as a binary file that represents each enrolled iris (the template), which requires huge bytes per eye to be stored (i.e., 307,200 bytes for a $640 \times 480$ grayscale image) [1]. This implicitly needs to minimize the size of image through the utilization of data compression techniques.

Image compression plays an essential role for effective transmission and storage of images [2]. The image compression systems are based on exploiting the redundancy within the image itself, along with the utilization of the limitations of human vision or perception [3]. Generally, they fall into two categories: lossless and lossy, depending on the redundancy(s) type(s) exploited [2]. Description of various lossless and lossy techniques can be found in previous reports [3-8].

During the last few years, several image compression techniques were developed in biometric systems such as the iris recognition system. Different types of image compression standards, including JPEG and JPEG-2000, were utilized to generate the compact iris data [2]. But, sometimes the lossless JPEG is not suitable for high compression ratios; another limitation is the blocking artifact that can occur at high compression ratio in case that the lossy-based system is used [2]. However, the challenge and necessity of improving efficient performance of biometric the iris compression system grew, since this data is increasing day by day, which becomes an increasingly intensive and important research area to design and adopt modern techniques [3].

Actually, most of the work on biometric iris sample data investigated the effects of image compression on recognition system performance, which clearly showed the inverse relation between the compression performance and recognition accuracy; when the compression rate increases, the recognition accuracy decreases, and vice versa [9]. Also, some efforts compared between different variants of lossless and lossy standard techniques (lossless: JPEG, JPEG-LS, PNG, GIF, JPEG2000, and JPEG XR) [10] and various extended forms of Discrete Wavelet Transform (DWT) that imply the Set Partitioning Into Hierarchical Tree $(S P I H)$ and Embedded Zero-Tree wavelet $(E Z W)$ coding techniques [11], along with curvelet transform in combination with lifting Wavelet [2]. The Region Of Interest (ROI) was also adopted to achieve highly compressed image quality [12]. Lastly, the hybrid technique was utilized efficiently as a mix between Lempel-Ziv-Welch (LZW) and Huffman coding [13].

The researchers exploited the Fourier transform as a tool to compress images in the last century, by exploiting the frequency domain image efficiently; each pixel represents a particular frequency contained in the spatial domain image, while compression occurs by modifying these coefficients. Some efforts aimed to improving the performance of the traditional Fourier transform-based technique, either by utilizing different variable block sizes instead of the fixed one [14], or using various quantization schemes of adaptive- or optimized-based techniques, where all the techniques still suffer from the existence of the imaginary embedded between the coefficients. Today, zipper transform is effectively in use, that exploits the conjugate symmetry property of Fourier transformation base to overcome the drawback of the traditional technique [15].

In this paper, an efficient method for compression biometric iris grayscale image is introduced that integrates the multiresolution scheme of DWT base along with the zipper transformation base techniques. The rest of the paper is organized as follows: sections 2, 3 and 4 contain comprehensive clarification of the traditional Fourier transform, zipper transform and the proposed system; the results for the proposed system are provided in section 5, and the conclusion in section 6 . 


\section{Iris Image Compression Using Traditional Discrete Fourier Transform Technique}

Basically, the Discrete Fourier transform (DFT) acts in mapping or transforming the input into coefficients using the linear combination of weighted basis function corresponding to the sine and cosine bases $[16,17]$. The implementation of the traditional discrete Fourier transform compression system is explained in the following steps [14-17].

Step 1: Load the input uncompressed iris gray image $I$ of $B M P$ format of size $N \times N$.

Step 2 Partition image $I$ into non-overlapped blocks (regions) to exploit the local similarity. Namely, the image needs to be partitioned into several smaller, non-overlapping blocks, that are either of fixed size $(n \times n)$ (i.e., $n<N)$ or of variable sizes $(n \times q)$ (i.e., $n<N \& q<N)$. For the first of these, the fixed partitioning method is adopted in this paper because of its simplicity and popularity. However, this occurs at the expense of efficiency and comes with a greater storage cost, because the blocks are partitioned based on the size of the region, regardless of the content and whether that region or block is uniform or non-uniform [3].

Step 3: Apply forward discrete Fourier transform on each segmented block according to the equation below.

Where $I(i, j)$ is the input correlated image of $i, j$ are the spatial coordinates and $u, v$ are coordinates in the transform domain. $F(u, v)$ is the transform coefficient at position $(u, v)$, where $u, v$, $i, j=0, \ldots \ldots . ., n-1$, also the base of the natural logarithmic function $e$ is about 2.71828; and $j_{\text {comp }}$ is the imaginary coordinate for a complex number, equals $\sqrt{-1}$.

Step 4: Apply symbol encoding techniques to remove the coding redundancy embedded within the Fourier coefficient's of complex nature.

Step 5: Reconstruct or rebuild the compressed or decoded image which is identical to the original one $I$, from the encoded coefficients, the decoder, simply use the inverse Fourier transform using the following equation:

\section{Iris Image Compression Using Zipper Transform Technique}

The core of zipper transform is identical to the Fourier transform, where this transformation coding-based technique aimed to decorrelate the input efficiently by utilizing the conjugate symmetry property of Discrete Fourier Transformation (DFT).

The technique simply starts from the original uncompressed iris image $I$, then implementing the discussed traditional Fourier transform coding method (DFT), where the output of DFT is a set of complex numbers having a property known as the conjugate symmetry, based on exploiting the interlacing and the concatenating zipper transform. The interlacing zipping operation is abstracted from the way and two separate entities can be fastened and tightened with a zipping tool. In the case of interlacing-ZT which corresponds to the forward transformation, the imaginary parts of the complex numbers in the upper half of the symmetry are stripped off and interlaced with their corresponding real counterparts that are subsequently encoded using Huffman coding techniques. In the concatenating $Z T$ (or simply ZT) on the other hand, the imaginary parts of the complex numbers in the upper half of the symmetry are stripped off and concatenated with their corresponding real counterparts that are corresponding to inverse transformation [15], followed by applying the IDFT to reconstruct the identical image $I$

\section{Iris Image Compression Using Double Transformation Technique}

In order to improve the performance of the zipper transform, which is an adaptive form of Fourier transform, the discrete wavelet transform (DWT) of Haar base of one multiresolution decomposition scheme was adopted. Due to less information being used compared to the original image (i.e., the use of a quarter part of the image instead of the whole image), the following steps were applied to compress iris image losslessly.

Step 1: Load the input uncompressed iris gray image $I$ of $B M P$ format of size $N \times N$.

Step 2: Perform a wavelet transform that decomposes $I$ image into four quadrants of approximation and detail sub bands ( $I_{L L}, I_{L H}, I_{H L}$ and $I_{H H}$, respectively), each has the size of $N / 2 \times N / 2$, where each sub band (approximation and details) was compressed differently. In other words, the $I_{L L}, I_{L H}, I_{H L}$ and $I_{H H}$ sub band partitions indicate the approximation image (average image) and the images with horizontal edges, vertical edges and diagonal edges, respectively.

Step 3: Apply the Zipper Transform of lossless base on the approximation $I_{L L}$, subband. 
Step 4: Encode the details $\left(I_{L H}, I_{H L}\right.$ and $I_{H H}$ ) subband losslessly using the symbol encoder of probability base.

Step 5: To reconstruct the decoded or compressed identical iris image $I$, the decoder reconstruct the approximation subband using the inverse transforms of zipper then then Fourier based respectively, and detailed sub bands coded information, with the inverse $D W T$.

\section{Experimental Results}

In order to test the performance of the traditional Fourier coding based techniques and comparing it with that of the zipper-based methods and the proposed double transformation-based technique, four standard gray ( 256 gray levels or 8 bits/pixel) square $(256 \times 256)$ images of highly details were utilized as shown in Figure-1. Also, the compression ratio was adopted as criteria of lossless system base (see equation 3), which is the ratio of the original image size to the compressed size in bytes. Here, the original image size corresponds to the size of the image represented by its width and height, resembling the number of rows and columns, respectively. The compressed size involves the encoding of the compressed size that is composed of size in bytes of DFT coefficients of real and imaginary parts (equation 4). Lastly, the size of the saved bytes corresponds to the difference between the original image size and the compressed image size (equation 5).

Comp.Ratio $=\frac{\text { Size of original image in bytes }}{\text { Size of compressed image in bytes }}$
Size of compressed image $=[$ Size in bytes for real image + Size in bytes for imageinary image $]$

Size of Saved Bytes $=[$ no.bytes for otiginal image - no.bytes for compressed image $]$

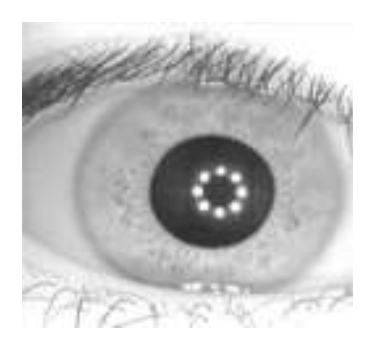

(a)

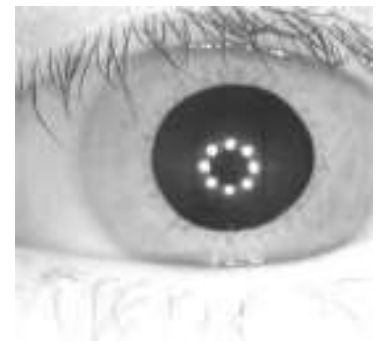

(b)

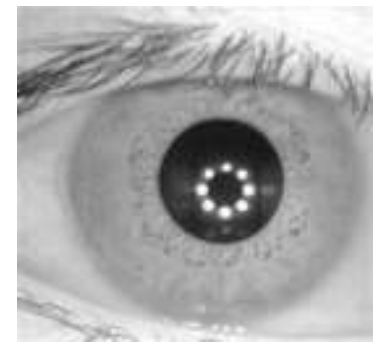

(c)

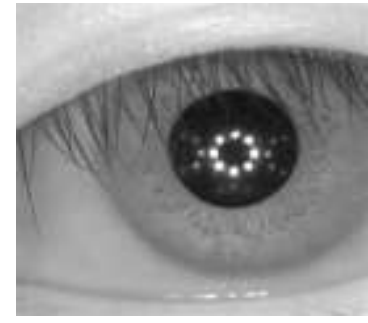

(d)

Figure 1-The four tested images of size 256×256, grayscale images, (a) Iris1, (b) Iris2, (c) Iris2 and (d) Iris4.

The results shown in Table-1 illustrate the comparison between the traditional Fourier transform, zipper transform and the proposed techniques, along with the utilization of the compression ratio and the size in bytes between the original and compressed images by using block size of $4 \times 4$. 
Table -1Comparison of the performance between traditional discrete Fourier transform, adaptive discrete Fourier transform of zipper base and double transformation of discrete wavelet transform using block size of $4 \times 4$ of tested images.

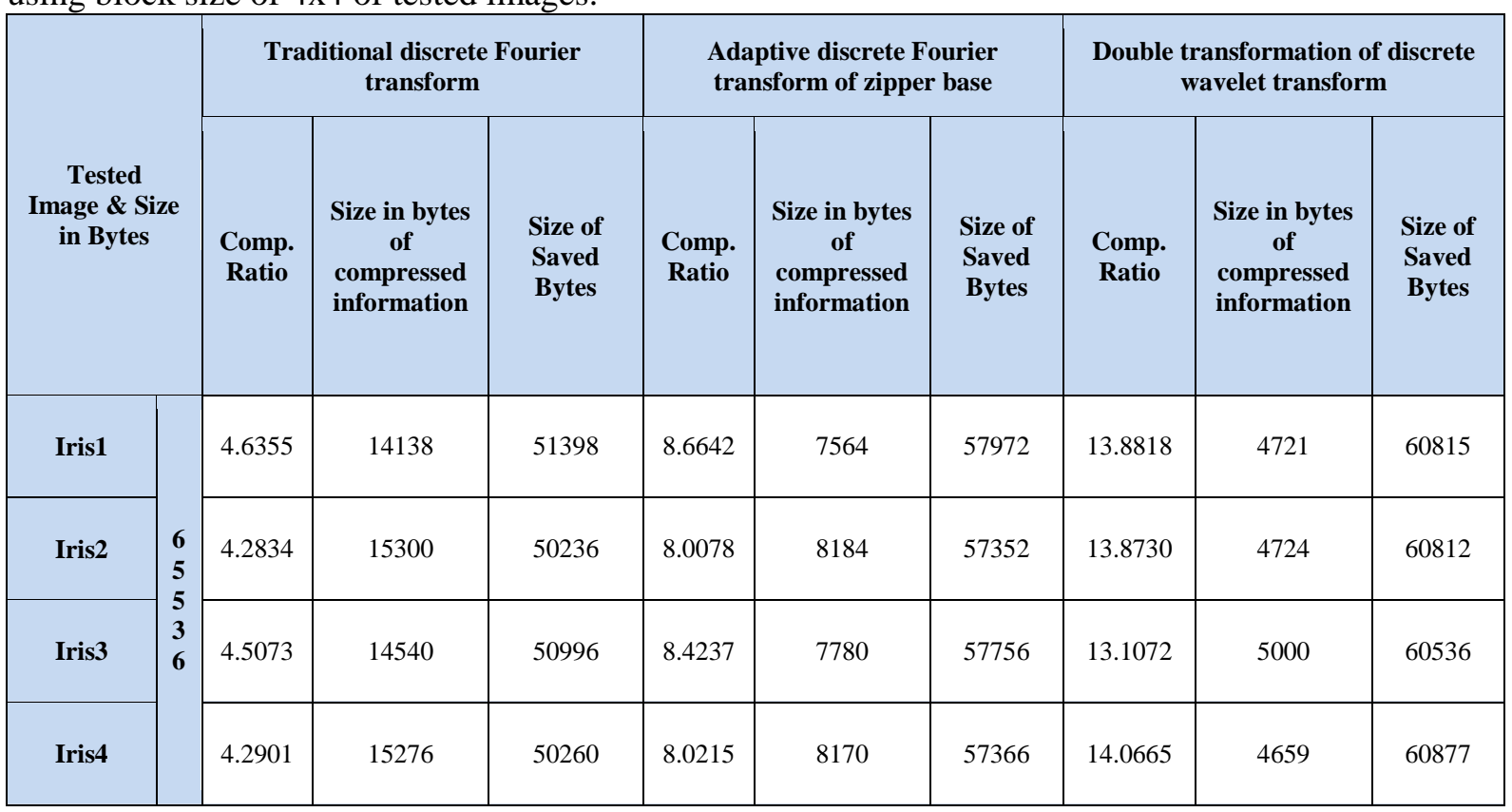

There is a number of issues that need to be highlighted according to the above results:

1- Obviously, the traditional Fourier compression system of the complex base provides lower compression ratio, since it would need greater range of values than the image in the spatial domain.

2- By utilizing zipper coding base, the higher compression ratio achieved, which was improved about twice in average as compared to the traditional Fourier coding for the tested images. This was due to the interlacing and the concatenating of complex coefficients.

3- It is clear that, by incorporating double transformation coding techniques, a higher compression ratio is attained due to the utilization of the decomposition base of approximation and detailed subbands.

Figure-2 demonstrates the performance of the traditional discrete Fourier transform of zipper base and double transformation base of the tested images using $4 \times 4$ block size.

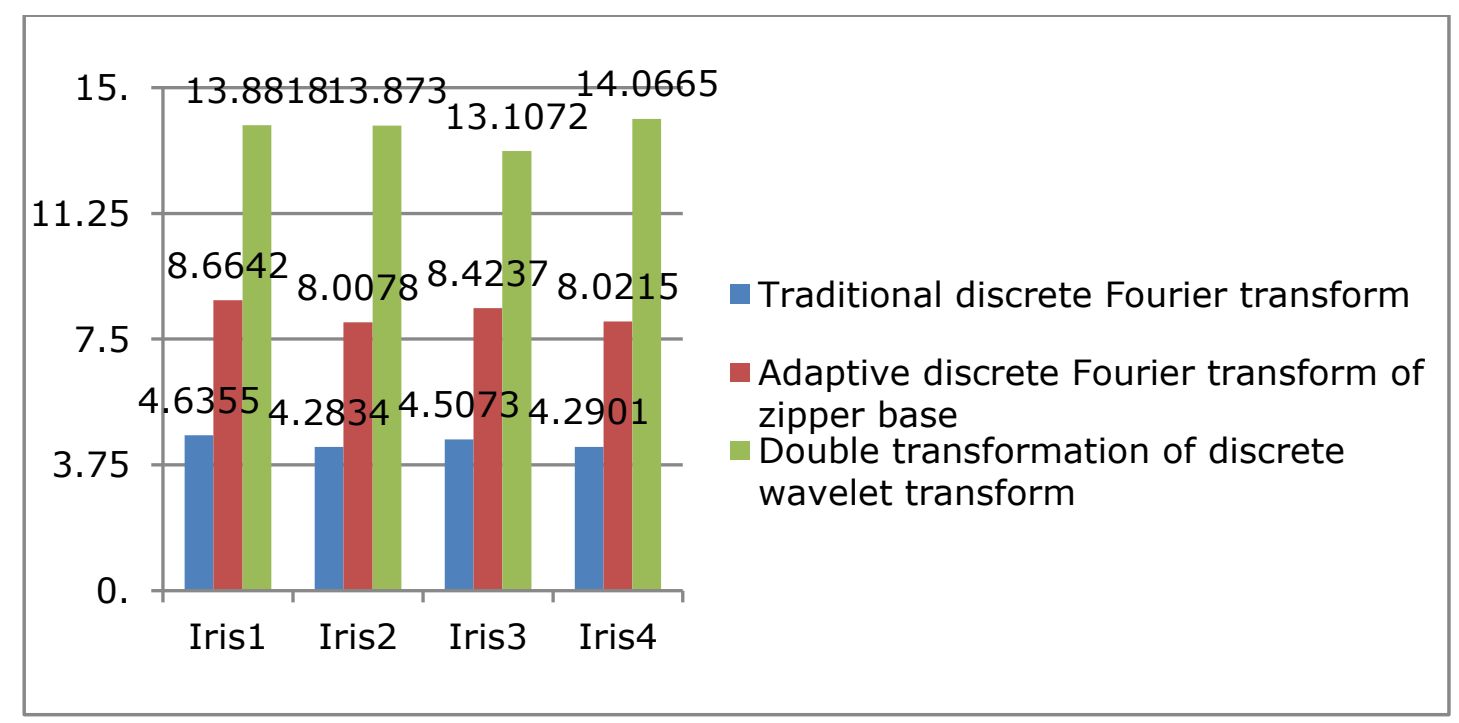

Figure 2-The performance of the traditional discrete Fourier transform, of zipper base and double transformation base of Iris 1, Iris2, Iris 3 and Iris 4 image using $4 \times 4$ block size. 


\section{Conclusions}

1) The traditional Fourier coding technique is characterized by simplicity, but on the expense of efficiency in terms of low compression ratio.

2) The adaptive discrete Fourier transform of zipper base efficiently improves the results, due to the exploitation of the interlacing and the concatenating in encoding and decoding processes, respectively. 3) The incorporation with the one-layered wavelet transforms of Haar base enhances the results, as achieved by the utilization of quarter part of the image as compared to the detailed subband.

\section{References}

1. Robert, W., Yingzi, D. and Daniel, A. 2009. Effects of Image Compression on Iris Recognition Performance and Image Quality, Conference: Computational Intelligence in Biometrics: Theory, Algorithms, and Applications, IEEE Xplore, CIB.2009.4925681, 2-7.

2. Saranya, D. and Annapoorani, P. 2016. Efficient Iris Image Compression Using Curvelet Transform. International Journal of Scientific Engineering and Applied Science (IJSEAS), 2(5): $410-413$.

3. Ghadah, Al-K. 2012. Intra and Inter Frame Compression for Video Streaming. Ph.D. thesis, Exeter University, UK.

4. 4-Puja, S. and Satyaranjan, P. 2014. A Survey of Image Compression Techniques. International Journal of Engineering and Innovative Technology, 4(2): 83-86.

5. Partishtha, G., Purohit, G. and Varsha, B. 2014. A Survey on Image Compression Techniques. International Journal of Advanced Reseach in Computer and Communication Engineering, 3(8): $7762-7768$.

6. Meenakshi, D. and Karthika Devi, V. 2015. Literature Review of Image Compression Techniques. International Journal of Computer Science \& Engineering Technology, 6(5): 286-288.

7. Hemalatha, M. and Nithya, S. 2016. A Through Survey on Lossy Image Compression Techniques. International Journal of Applied Engineering Research, 11(5): 3326-3329.

8. Akhilesh, K. and Malviya, A. 2017. A Survey on Image Compression Methods. International Journal of Engineering and Computer Science, 6(5): 21393-21400.

9. Weinhandel, G., Stögner, H. and Uhl, A. 2009. Experimental study on lossless compression of biometric sample data. In Proceedings of the 6th International Symposium on Image and Signal Processing and Analysis, Salzburg, Austria, 1 - 6.

10. Kurt, H., Herbert, S., Andreas, U. and Georg, W. 2011. Lossless Compression of Polar Iris Image Data, Iberian Conference on Pattern Recognition and Image Analysis, Springer, Berlin, Heidelberg, 6669, 329 - 337.

11. Shoba, K. and Pushkar, M. 2013. Comparison of IRIS Image Compression using JPEG 2000 and SPIHT algorithm. Journal of Electronics and Communication Engineering (IOSR-JECE), 4(4): 5 9.

12. Shaikh, I. and Mukane, S. 2015. Iris Image Compression Using JPEG \& Its Effect on Recognition Performance, International Journal of Scientific \& Engineering Research, 6(2): 1093 - 1098.

13. Sangeetha, M., Betty, P. and Nanda, K. 2017. A Biometrie Iris Image Compression Using LZW and Hybrid LZW Coding Algorithm. International Conference on Innovations in Information, Embedded and Communication Systems (ICIIECS), Coimbatore, India, IEEE Xplore, 1- 6.

14. Kanaka, M., Haragopal, V. and Jyothi, S. 2016. Statistical Image Compression Using Fast Fourier Coefficients, International Journal of Computer Applications, 155(3): 31 -36.

15. Babajide, O. 2017. A Fast and Efficient Near-Lossless Image Compression Using Zipper Transformation, Electrical and Computer Engineering University of Louisville, Louisville, KY 40218, 1 - 13..

16. Gonzalez, R. C. and Woods, R. E. 2003. Digital Image Processing. 2nd ed. Prentice Hall.

17. Shi, Y. Q. and Sun, H. 2000. Image and Video Compression for Multimedia Engineering. CRC Press, London. 